\title{
PRODUCTS AND REMOTE POINTS: EXAMPLES AND COUNTEREXAMPLES
}

\author{
A. DOW AND T. J. PETERS \\ (Communicated by Doug Curtis)
}

\begin{abstract}
Examples of products with remote points and counterexamples of products without remote points are given. The paradoxical behavior of remote points with respect to products is exhibited.

Also, an example is given of spaces $X$ and $Y$, where neither $X$ nor $Y$ has a $\sigma$-locally finite $\pi$-base, but $X \times Y$ does.
\end{abstract}

1. Introduction. The literature is rich with theorems proving the existence of remote points for various products $\left[\mathbf{C S}, \mathbf{v D}, \mathbf{D}_{\mathbf{1}}, \mathbf{D}_{\mathbf{2}}, \mathbf{D}_{\mathbf{3}}, \mathbf{D}_{\mathbf{4}}, \mathbf{D P}, \mathbf{H P}, \mathbf{v M}\right.$, $\mathbf{P}_{\mathbf{1}}, \mathbf{P}_{\mathbf{2}}, \mathbf{P}_{\mathbf{3}}$ ] and with counterexamples showing the absence of remote points for specific products $\left[\mathbf{v D v M}, \mathbf{D}_{\mathbf{5}}, \mathbf{D}_{\mathbf{6}}, \mathbf{P}_{\mathbf{1}}\right]$. However, there are still some questions remaining about the relationship between products and remote points.

This work bounds some of the extremes of this situation by providing two unusual and complementary example-counterexample pairs.

The notation $\left(\mathrm{ZFC}^{+}\right)$indicates that the proof utilizes a set theoretic assumption that is not contained within ZFC.

The first pair is motivated by the following considerations. It is known that there are spaces $X$, such that $\omega \times X$ has no remote points (e.g., see [vDvM]). One naturally wonders if it is possible for there to be an $X$ such that $\kappa \times X$ has no remote points for any $\kappa$ (by the example below, it is consistent that this cannot happen). Similarly, it is natural to ask whether there is a $\kappa$ large enough to guarantee that $\kappa \times X$ has remote points for any $X$. (The following counterexample provides a negative answer.)

The first pair consists of the following:

EXAMPLE $\left(\mathrm{ZFC}^{+}\right)$. For each $X$, there exists a cardinal $\kappa$ such that $\kappa \times X$ has remote points.

COUNTEREXAMPLE. For each cardinal $\kappa$, there exists a space $X$ such that $\kappa \times X$ has no remote points.

The second pair is motivated by the extremely natural question, "Is the property of having remote points productive?" There are many partial affirmative responses to this question $\left[\mathbf{D}_{\mathbf{1}}, \mathbf{D}_{\mathbf{4}}, \mathbf{D P}, \mathbf{H P}\right.$, and $\left.\mathbf{P}_{\mathbf{3}}\right]$. Thus, it is rather surprising that this

Received by the editors April 21, 1987 and, in revised form, November 24, 1987 and February 29, 1988. The contents of this paper were presented by the second author at the Spring Topology Conference, Gainseville, Florida, 1988.

1980 Mathematics Subject Classification (1985 Revision). Primary 54D40, 54D35, 54B10, 03E35, 03E55; Secondary 54A25, 54B25, 54G20.

Key words and phrases. $\beta X, \sigma$-locally finite $\pi$-base, $\sigma$ - $\pi$ space, $\pi$-base, infinitary combinatorics, nonpseudocompact, product, remainder, remote points, Stone-Cech compactification.

The first author thanks NSERC of Canada for financial assistance and the second author thanks Computervision Corporation for financial assistance. 
work shows that it is consistent that the property of having remote points is not productive. Additionally, it is shown that a product may have remote points even when neither of its nonpseudocompact factors has remote points.

The second pair consists of the following:

EXAMPLE. There exist nonpseudocompact spaces $X$ and $Y$, each having no remote points, such that $X \times Y$ has remote points. (Furthermore, neither $X$ nor $Y$ has a $\sigma$-locally finite $\pi$-base, but $X \times Y$ does.)

COUNTEREXAMPLE $\left(\mathrm{ZFC}^{+}\right)$. There exist nonpseudocompact spaces $X$ and $Y$, each having remote points, such that $X \times Y$ has no remote points.

These two example-counterexample pairs demonstrate the complexity of the situation with respect to remote points for products.

In the first example-counterexample pair, the discrete factor always has remote points, whereas the product may or may not.

In the second example-counterexample pair, the product may or may not have remote points irrespective of whether the nonpseudocompact factors do. (For logical completeness, this last statement involves four cases. Only two cases are presented in this paper, because the other two are well known. That is, within the class of nonpseudocompact spaces, there exist factors with remote points whose product has remote points $\left[\mathbf{C S}, \mathbf{v D}, \mathbf{D}_{\mathbf{1}}, \mathbf{D P}, \mathbf{H P}, \mathbf{v M}, \mathbf{P}_{\mathbf{1}}, \mathbf{P}_{\mathbf{2}}, \mathbf{P}_{\mathbf{3}}\right]$ and there exist factors with no remote points whose product has no remote points $\left[\mathbf{D}_{\mathbf{5}}\right]$.)

2. Preliminaries. All spaces considered will be assumed to be completely regular Hausdorff.

2.1 Definition. For a space $X$, a point $p \in \beta X \backslash X$ is remote if $p \notin \operatorname{cl}_{\beta X} D$ for any nowhere dense subset $D$ of $X$.

2.2 DEFINITION. A space with a $\sigma$-locally finite $\pi$-base will be called a $\sigma$ - $\pi$ space.

2.3 DEFinition. For a space $X$ and $\mathscr{F}$ a collection of subsets of $X$, the collection $\mathscr{F}$ is said to be remote if for each nowhere dense $D \subset X$, there exists an $F_{D} \in \mathscr{F}$ such that $D$ and $F_{D}$ are completely separated. ${ }^{1}$

2.4 DEFinition. The cellularity of a space $X$, abbreviated $\mathrm{c} X$, is the smallest cardinal $\alpha$ for which each pairwise disjoint family of nonempty open subsets of $X$ has $\alpha$ or fewer elements.

2.5 Definition. For a space $X$, if $\mathrm{c} X=\omega$, then $X$ has the countable chain condition. The abbreviation ccc indicates that a space has the countable chain condition.

2.6 Definition. A compact Souslin line is a compact, connected, linearly ordered space with topology defined by its order, which is ccc but not separable.

For a set $A$, the notation $|A|$ will indicate the cardinality of $A$. For a space $X$, the notation $\pi X$ will denote the $\pi$-weight of $X$. For a cardinal $\kappa$, the notation $c f(\kappa)$ will denote the cofinality of $\kappa$. Whenever a cardinal is used as a topological space, it will be assumed to have the discrete topology. For an arbitrary set $S$ and a cardinal $\kappa$, let

$$
[S]^{\kappa}=\{A: A \subset S \text { and }|A|=\kappa\} .
$$

For sets $S$ and $T$, and a function $f: S \rightarrow T$, the notation $f: S \rightarrow T$ indicates that $f$ is onto $T$.

\footnotetext{
${ }^{1}$ Dow [ $\mathbf{D}_{1}$ ], in context of closed subsets of normal spaces defined remote collections by only requiring that $D$ and $F_{D}$ be disjoint. The definition given above is the generalization for the class of completely regular Hausdorff spaces.
} 
3. Example with remote points. If the following set theoretic assumption was false in a model of ZFC, then there would exist a model with a measurable cardinal. $^{2}$

3.1 Assumption. There exist arbitrarily large $\kappa$, with $2^{\kappa}=\kappa^{+}$.

Throughout $\S 3$, it will be understood that the model used satisfies Assumption $3.1 .^{3}$

Let $X$ be a space and let $\kappa$ be a cardinal with $2^{\kappa}=\kappa^{+}$, such that $\kappa \geq \pi X$.

Let $B$ be a $\pi$-base for $\beta X$, where it is assumed, without loss of generality, that $\beta X \in B$, and $|B| \leq \kappa$.

Let $\mathscr{C}$ be the set of those maximal cellular families of $\kappa \times \beta X$ such that if $c \in C \in \mathscr{C}$, then $c=\{\alpha\} \times b$ for some $\alpha<\kappa$ and some $b \in B$.

Note that $\mathscr{C} \subset \mathscr{P}(\kappa \times B)$ and that $|\mathscr{P}(\kappa \times B)| \leq 2^{\kappa}=\kappa^{+}$(where the last equality follows because of set theoretic Assumption 3.1). Therefore it follows that on $\kappa \times \beta X$, there exists $\kappa^{+}$-many nowhere dense sets, say $\left\{D_{\xi}: \xi<\kappa^{+}\right\}$, such that each of nowhere dense set is contained in some $D_{\xi}$.

3.2 LEMMA. For each $\xi<\kappa^{+}$, there exists $U_{\xi}$, a nonempty open subset of $\kappa \times \beta X$, such that

(i) $\left[\mathrm{cl}_{\kappa \times \beta X} U_{\xi}\right] \cap D_{\xi}=\varnothing$, and

(ii) if $n<\omega$, and $\xi_{1}, \ldots, \xi_{n} \leq \xi$, then $\left|\left\{\alpha<\kappa: \bigcap_{i=1}^{n} U_{\xi_{i}} \cap(\{\alpha\} \times \beta X) \neq \varnothing\right\}\right|$ $=\kappa$.

PROOF. The proof is by transfinite induction on $\xi$. Suppose $U_{\gamma}$ exists for each $\gamma<\xi$. Let $\mathscr{U}$ be the family of all finite nonempty intersections of the $U_{\gamma}$ 's. Let $\mathscr{U}=\left\{V_{\lambda}: \lambda<\kappa\right\}$. For each $V_{\lambda}, \lambda<\kappa$, recursively choose $\alpha_{\lambda}<\kappa$ such that $V_{\lambda} \cap\left(\left\{\alpha_{\lambda}\right\} \times \beta X\right) \neq \varnothing$, and such that if $\lambda^{\prime}<\lambda$, then $\alpha_{\lambda^{\prime}}<\alpha_{\lambda}$. Then, inside each $V_{\lambda} \cap\left(\left\{\alpha_{\lambda}\right\} \times \beta X\right)$, choose a nonempty open subset $W_{\lambda}$ such that $\operatorname{cl}_{\kappa \times \beta X} W \cap D_{\xi}=\varnothing$. Let $U_{\xi}=\bigcup_{\lambda<\kappa} W_{\lambda}$.

3.3 EXAMPLE $\left(\mathrm{ZFC}^{+}\right)$. For each $X$, there exists a cardinal $\kappa$ such that $\kappa \times X$ has remote points.

PrOOF. Let $\kappa \geq \pi X$ such that $2^{\kappa}=\kappa^{+}$.

Let $\mathscr{G}=\left\{\operatorname{cl}_{\kappa \times \beta X} U_{\xi}: \xi<\kappa^{+}\right\}$, where $U_{\xi}$ is as described in Lemma 3.2. Choose any $p \in \beta(\kappa \times \beta X) \backslash(\kappa \times \beta X)$ such that $p \in \operatorname{cl}_{\beta(\kappa \times \beta X)} G$ for all $G \in \mathscr{G}$. It is easily verified that $p$ is a remote point of $\kappa \times \beta X$. But, the existence of remote points for $\kappa \times \beta X$ is sufficient to imply the existence of remote points for $\kappa \times X\left[\mathbf{P}_{1}, 4.3 ; \mathbf{P}_{2}\right.$, 4.11].

3.4 REMARK. Although the set theoretic assumption 3.1 is very weak, it would still be very interesting to see whether it can be avoided. Does there exist a cardinal function $\kappa$ such that for any space $X$, the space $\kappa(\pi X) \times X$ has remote points? It is known that $\kappa(\omega)=\omega[\mathbf{C S}, \mathbf{v D}]$, but not even the value (if any exists) of $\kappa\left(\omega_{1}\right)$ is unknown.

\footnotetext{
${ }^{2}$ The statement follows from the implication: Con (there exists a strong limit cardinal $\kappa$, with $\operatorname{cf}(\kappa)=\omega$ and $\left.2^{\kappa}>\kappa^{+}\right) \Rightarrow$ Con (there exists a model with a measurable cardinal) [KM, p. 261]).

${ }^{3}$ Since set theoretic assumptions are a matter of individual taste, it is worthwhile to note that the much stronger (but more conventional) assumption of GCH could be used in place of Assumption 3.1 .
} 
4. Counterexample with no remote points. This section utilizes only ZFC.

There exists a large class of spaces of cellularity greater than $\omega_{1}$ which have no remote points $\left[\mathbf{D}_{\mathbf{5}}\right]$. By means of minor technical changes, this result can be generalized to exhibit the counterexample for this section. First, the result from $\left[\mathbf{D}_{\mathbf{5}}\right]$ is stated.

4.1 THEOREM $\left[\mathbf{D}_{5}\right]$. If $Y$ is a compact space and $c Y>\omega_{1}$, then $\omega \times Y^{\omega}$ has no remote points.

Since the proof of the above result is quite long and since the technical modifications are minor, the generalization will be stated without proof.

4.2 THEOREM. If $Y$ is compact and $c Y>\alpha^{+}$, then the space $\gamma \times Y^{\beta}$ has no remote points, for any $\gamma \leq \alpha$ and for any $\beta \geq \omega$.

4.3 COUNTEREXAMPLE. For each cardinal $\kappa$, there exists a space $X$ such that $\kappa \times X$ has no remote points.

ProOF. Let $Y$ be a compact space such that $\mathrm{c} Y>\kappa^{+}$. Let $X=Y^{\omega}$.

4.4 COMMENT. Let us review the situation for the existence of remote points of $\kappa \times X$, when $2^{\kappa}=\kappa^{+}$. In $\S 3$, it was shown that if $\pi X \leq \kappa$, then $\kappa \times X$ has remote points. In fact, the same proof is applicable to the situation where $c X \leq \kappa$ and $\pi X \leq \kappa^{+}$. For the special case of $\kappa=\omega$, a proof similar to that of $\left[\mathbf{D}_{4}, 2.5\right]$ can be used to show that $\omega \times X$ has remote points whenever $\pi X \leq \omega_{1}$. Conversely, in Counterexample 4.3 , it is shown that $\kappa \times X$ can have no remote points, in cases where $\pi X \leq \kappa^{++}$-thus preventing any further generalization of those preceding affirmative results where $\pi$-weight is the only consideration. However, very little is known, even for $\kappa=\omega$, about the case where $c X \leq \kappa$ and $\pi X>\kappa^{+}$.

5. Example with remote points. This section utilizes only ZFC.

For spaces $X$ and $Y$, and a continuous function $f: X \rightarrow Y$, the notation $\beta f$ will indicate the Stone extension of $f$. The notation $U\left(\omega_{1}\right)$ denotes the space of uniform ultrafilters on $\omega_{1}$, that is

$$
U\left(\omega_{1}\right)=\left\{p \in \beta \omega_{1}:|A|=\omega_{1} \text { for all } A \in p\right\} .
$$

Since $U\left(\omega_{1}\right)$ can be imbedded in $\beta c^{+} \backslash c^{+}$, it will be assumed, without loss of generality, that $U\left(\omega_{1}\right) \subset \beta c^{+} \backslash c^{+}$. For any function $f$, the notation $\operatorname{dom}(f)$ indicates the domain of $f$.

5.1 Definition. Let $p \in U\left(\omega_{1}\right)$, and choose $q \in U\left(\omega_{1}\right)$ such that $p$ and $q$ are not type-equivalent (relative to $U\left(\omega_{1}\right)$ ). Let $\tau(p)$ be the type of $p$ (relative to $\left.U\left(c^{+}\right)\right)$. Let $X_{p}=c^{+} \cup \tau(p)$. Similarly, define $X_{q}$.

Because of the similarity of the definitions of $X_{p}$ and $X_{q}$, Lemmas 5.4-5.7 below will only be proven for the case of $p$, but it will be clear that this is sufficient.

5.2 REMARK. The spaces $X_{p}$ and $X_{q}$ are nonpseudocompact.

5.3 REMARK. For each $s \in \bigcup_{n<\omega}{ }^{n} c^{+}$, let $[s]=\left\{x \in X_{p}^{\omega}: s(i)=x_{i}, i \in \operatorname{dom}(s)\right\}$. Let $\mathscr{B}=\left\{[s]: s \in \bigcup_{n<\omega}{ }^{n} c^{+}\right\}$. It is clear that $\mathscr{B}$ is a $\pi$-base for $X_{p}^{\omega}$. (A similar $\pi$-base exists for $X_{q}^{\omega}$.)

Recall the following definitions $\left[\mathrm{CN}_{2}\right.$, p. 22]. For $\alpha \geq \beta, X$ is pseudo- $(\alpha, \beta)$ compact if for every set $\left\{U_{\xi}: \xi<\alpha\right\}$ of nonempty open subsets of $X$, there is $x \in X$ 
such that

$$
\left|\left\{\xi<\alpha: V \cap U_{\xi} \neq \varnothing\right\}\right| \geq \beta
$$

for every neighborhood $V$ of $x$.

A pseudo- $(\alpha, \omega)$-compact space is said to be pseudo- $\alpha$-compact.

\subsection{LEMMA. The spaces $X_{p}, X_{q}, X_{p}^{\omega}$, and $X_{q}^{\omega}$ are pseudo- $\omega_{1}$-compact.}

PROOF. Suppose $\left\{s_{\alpha}: \alpha<\omega_{1}\right\} \subset<\omega^{+}$. Since for each $\alpha<\omega_{1}$, there exists $n_{\alpha}<\omega$ such that $s_{\alpha} \in{ }^{n_{\alpha}} c^{+}$, it follows that there exist $n<\omega$ and $I_{0} \in\left[\omega_{1}\right]^{\omega_{1}}$ such that $s_{\alpha} \in{ }^{n} c^{+}$for all $\alpha \in I_{0}$.

For $m<\omega$, recursively define $I_{m+1}$ such that $I_{0} \supset I_{1} \supset \cdots \supset I_{m+1} \supset \cdots$; $\left|I_{m+1}\right|=\omega_{1}$, and for each $0 \leq j<m$, either

Case 1. There exists $\gamma_{j} \in c^{+}$such that $s_{\alpha}(j)=\gamma_{j}$ for all $\alpha \in I_{j+1}$ or

Case 2. For $\alpha, \beta \in I_{j+1}$ with $\alpha<\beta, s_{\alpha}(j) \neq s_{\beta}(j)$.

For each $k<\omega$, define $r_{k} \in X_{p}$ as follows. If $k<n$ and Case 1 holds, let $r_{k}=\gamma_{k}$. If $k<n$ and Case 2 holds, then define $f_{k}: I_{n+1} \stackrel{1-1}{\rightarrow} c^{+}$such that $f_{k}(\alpha)=s_{\alpha}(k)$ for all $\alpha \in I_{n+1}$. Also, define $g: \omega_{1} \stackrel{1-1}{\rightarrow} I_{n+1}$ arbitrarily. Let $r_{k}=\beta\left(f_{k} \circ g\right)(p)$ and note that $r_{k}=\beta f_{k}(\beta g(p))$. For $k \geq n$, choose $r_{k} \in X_{p}$ arbitrarily.

Let $r=\left(r_{0}, r_{1}, \ldots\right)$. Then $r \in X_{p}^{\omega}$.

Let $J=J_{0} \times J_{1} \times \cdots \times J_{n} \times X_{p} \times X_{p} \times \cdots$ be a neighborhood of $r$.

In order to show that $X_{p}^{\omega}$ is pseudo- $\left(\omega_{1}, \omega_{1}\right)$-compact, it suffices to show, for each $k<n$, that $\left\{\alpha \in I_{n+1}: s_{\alpha}(k) \in J_{k}\right\} \in \beta g(p)$.

Note that for each $k<n, A \in r_{k}$ if and only if $f_{k}^{-1}(A) \in \beta g(p)$. Therefore, for each $k<n,\left\{\alpha: s_{\alpha}(k) \in J_{k}\right\}=f_{k}^{-1}\left(J_{k} \cap c^{+}\right) \in \beta g(p)$, as was to be shown.

Hence, $X_{p}^{\omega}$ is pseudo- $\left(\omega_{1}, \omega_{1}\right)$-compact, which implies that $X_{p}^{\omega}$ is pseudo- $\omega_{1}$ compact $\left[\mathbf{C N}_{2}, 2.1\right]$. Hence, $X_{p}$ is also pseudo- $\omega_{1}$-compact.

\subsection{LEMMA. The space $X_{p}$ is not $\sigma-\pi$.}

ProOF. Since the $\pi$-weight of $X_{p}^{\omega}$ is $c^{+}$and $X_{p}^{\omega}$ is pseudo- $\omega_{1}$-compact, $X_{p}^{\omega}$ is not $\sigma-\pi\left[\mathbf{P}_{\mathbf{3}}, 3.5 \mathrm{a}\right]$.

5.6 LEMMA. Let $\mathscr{A} \subset \mathscr{B}$ such that $\mathscr{A}$ is maximal cellular, let $F$ be a regular closed subset of $X_{p}^{\omega}$ such that $F \subseteq \bigcup \mathscr{A}$, and let $\mathscr{A}(F)=\{[s] \in \mathscr{A}:[s] \cap F \neq \varnothing\}$. Then $\mathscr{A}(F)$ is countable.

Proof. Since $\operatorname{int}(F)$ is dense in $F$, we may choose $\left[t_{s}\right] \subseteq[s] \cap F$ for each $[s] \in \mathscr{A}(F)$. Clearly each point of $\bigcup \mathscr{A}$ has a neighborhood meeting at most one member of $\left\{\left[t_{s}\right]:[s] \in \mathscr{A}(F)\right\}$. Also $\operatorname{cl} \bigcup\left\{\left[t_{s}: s\right] \in \mathscr{A}(F)\right\} \subseteq F \subseteq \bigcup \mathscr{A}$, hence $\left\{\left[t_{s}\right]:[s] \in \mathscr{A}(F)\right\}$ is locally finite. Since $X_{p}^{\omega}$ is pseudo- $\omega_{1}$-compact, $\mathscr{A}(F)$ is countable.

\subsection{LEMMA. $X_{p}^{\omega}$ and $X_{q}^{\omega}$ have no remote points.}

ProOF. Assume that $X_{p}^{\omega}$ has remote points. Hence, there must exist a ultrafilter $\mathscr{F}$ of regular closed subsets of $X_{p}^{\omega}$ such that for each nowhere dense subset $D$ of $X$, there exists $F_{D} \in \mathscr{F}$ with $F_{D} \cap D=\varnothing$.

Let $S=\left\{\alpha<c^{+}: \operatorname{cf}(\alpha)=\omega_{1}\right\}$ and note that $S$ is stationary [K]. For each $\alpha \in S, \alpha^{\omega}$ is nowhere dense and there exists a maximal cellular family $\mathscr{A}_{\alpha} \subset \mathscr{B}$ such that $\alpha^{\omega} \subset X_{p}^{\omega} \backslash \cup \mathscr{A}_{\alpha}$. Furthermore, for each $\alpha \in S$, there exists $F_{\alpha} \in \mathscr{F}$ such 
that $F_{\alpha} \subset \bigcup \mathscr{A}_{\alpha}$. For each $\alpha \in S$, let

$$
\mathscr{A}_{\alpha}\left(F_{\alpha}\right)=\left\{\left[s_{n}^{\alpha}\right]: n<\omega\right\}
$$

and note that $F_{\alpha} \subset \cup \mathscr{A}_{\alpha}\left(F_{\alpha}\right)$.

Without loss of generality, it may be assumed that for each $\alpha \in S$ and each $n<\omega$, there may be chosen $k_{n}^{\alpha}<\omega$ such that $s_{n}^{\alpha} \in k_{n}^{\alpha}+1 c^{+},\left.s_{n}^{\alpha}\right|_{k_{n}^{\alpha}} \in{ }^{k_{n}^{\alpha}} \alpha$, and $s_{n}^{\alpha}\left(k_{n}^{\alpha}\right) \geq \alpha$. Define $f_{0}: S \rightarrow c^{+}$as follows. For each $\alpha \in S$,

$$
f_{0}(\alpha)=\sup _{\substack{n<\omega \\ j<k_{n}^{\alpha}}}\left\{\lambda: s_{n}^{\alpha}(j)=\lambda\right\} .
$$

For each $\alpha \in S$, note that $f_{0}(\alpha)<\alpha$, since $c f(\alpha)=\omega_{1}$. Hence, there exists $\gamma<c^{+}$ such that $f_{0}^{-1}(\gamma)$ is stationary $[\mathbf{K}, 6.15]$. Let $S_{0}=\left(\gamma, c^{+}\right) \cap f_{0}^{-1}(\gamma)$, then $S_{0}$ is stationary and $S_{0} \subset S$.

Let $\left\{\left\{t_{i}: i<\omega\right\}_{\xi}: \xi<c\right\}$ index all countable sequences such that for each $i<\omega$, $t_{i} \in \bigcup_{n<\omega}^{n} \gamma$, where it should be noted that $|\gamma| \leq c$.

Define $f_{1}: S_{0} \rightarrow c$ as follows. For each $\alpha \in S_{0}, f_{1}(\alpha)=\xi$, where $\xi<c$ is chosen such that

$$
\left\{t_{i}: i<\omega\right\}_{\xi}=\left\{\left.s_{n}^{\alpha}\right|_{k_{n}^{\alpha}}: n<\omega\right\} .
$$

For each $\alpha \in S_{0}$, note that $f_{1}(\alpha)<c<\alpha$ (i.e., $f_{1}(\alpha)<\alpha$ ). Hence, there exists $\xi^{*}<c$ such that $f_{1}^{-1}\left(\xi^{*}\right)$ is stationary. Let $S_{1}=f_{1}^{-1}\left(\xi^{*}\right)$, where $S_{1} \subset S_{0} \subset S$. Note that if $\alpha \in S_{1}$, then

$$
\left\{\left.s_{n}^{\alpha}\right|_{k_{n}^{\alpha}}: n<\omega\right\}=\left\{t_{i}: i<\omega\right\}_{\xi^{*}} .
$$

Choose $\alpha^{*} \in S_{1}$, and choose $\beta^{*} \in S_{1}$ subject to the conditions:

(i) $\beta^{*} \in S_{1} \backslash \alpha^{*}$,

(ii) $\left\{s_{n}^{\alpha^{*}}: n<\omega\right\} \subset \bigcup_{n<\omega}{ }^{n} \beta^{*}$.

These conditions may be simultaneously satisfied because of the cofinality of $S_{1}$ in $c^{+}$.

Recall, from the beginning of the proof, that $F_{\alpha^{*}}, F_{\beta^{*}} \in \mathscr{F}, F_{\alpha^{*}} \subset \bigcup_{n<\omega}\left[s_{n}^{\alpha^{*}}\right]$, and $F_{\beta^{*}} \subset \bigcup_{n<\omega}\left[s_{n}^{\beta^{*}}\right]$. A contradiction to $\mathscr{F}$ being an ultrafilter will now be obtained by showing that for any $m, n<\omega$,

$$
\left[s_{n}^{\alpha^{*}}\right] \cap\left[s_{m}^{\beta^{*}}\right]=\varnothing \text {. }
$$

Case 1. $k_{n}^{\alpha^{*}}<k_{m}^{\beta^{*}}$. Clearly, $s_{n}^{\alpha^{*}}\left(k_{n}^{\alpha^{*}}\right) \in\left[\alpha^{*}, \beta^{*}\right)$. However, $s_{m}^{\beta^{*}}\left(k_{n}^{\alpha^{*}}\right)<\gamma$, because $\beta^{*} \in S_{0}$. Furthermore, $\gamma<\alpha^{*}$, since $\alpha^{*} \in S_{0}$. Thus, $s_{m}^{\beta^{*}}\left(k_{n}^{\alpha^{*}}\right)<\gamma<\alpha^{*} \leq$ $s_{n}^{\alpha^{*}}\left(k_{n}^{\alpha^{*}}\right)$.

Case 2. $k_{n}^{\alpha^{*}}=k_{m}^{\beta^{*}}$. Again, $s_{n}^{\alpha^{*}}\left(k_{n}^{\alpha^{*}}\right) \in\left[\alpha^{*}, \beta^{*}\right)$. However, $s_{m}^{\beta^{*}}\left(k_{n}^{\alpha^{*}}\right)=s_{m}^{\beta^{*}}\left(k_{m}^{\beta^{*}}\right)$ $\geq \beta^{*}$. Thus $s_{n}^{\alpha^{*}}\left(k_{n}^{\alpha^{*}}\right)<\beta^{*} \leq s_{m}^{\beta^{*}}\left(k_{n}^{\alpha^{*}}\right)$.

Case 3. $k_{n}^{\alpha^{*}}>k_{m}^{\beta^{*}}$. Then $s_{n}^{\alpha^{*}}\left(k_{m}^{\beta^{*}}\right)<\alpha^{*}$, since $\alpha^{*} \in S$. However, $s_{m}^{\beta^{*}}\left(k_{m}^{\beta^{*}}\right) \geq \beta^{*}$, since $\beta^{*} \in S$. Thus, $s_{n}^{\alpha^{*}}\left(k_{m}^{\beta^{*}}\right)<\alpha^{*}<\beta^{*} \leq s_{m}^{\beta^{*}}\left(k_{m}^{\beta^{*}}\right)$.

These three cases having been demonstrated, the proof is now complete.

5.8 LEMMA. The product $X_{p} \times X_{q}$ is not pseudo-c ${ }^{+}$-compact.

Proof. Suppose $X_{p} \times X_{q}$ is pseudo- $c^{+}$-compact. Then the family $\{(\alpha, \alpha): \alpha<$ $\left.c^{+}\right\}$of open subsets of $X_{p} \times X_{q}$ must have a limit point in $X_{p} \times X_{q}$. Let $\left(r_{0}, r_{1}\right)$ represent such a limit point, where $r_{0}$ is type-equivalent to $p$ and $r_{1}$ is type-equivalent to $q$. 
Recall that $p$ is not type-equivalent to $q$. Hence, there must exist $A_{0} \in r_{0}$ and $A_{1} \in r_{1}$ such that $A_{0} \cap A_{1}=\varnothing$. However, then $A_{0} \times A_{1} \cap\left\{(\alpha, \alpha): \alpha<c^{+}\right\}=\varnothing$ contradicts that $\left(r_{0}, r_{1}\right)$ is a limit point of $X_{p} \times X_{q}$, and the proof is complete.

5.9 LEMMA. The product $X_{p}^{\omega} \times X_{q}^{\omega}$ is a $\sigma-\pi$ space. $^{4}$

ProOF. The product $X_{p}^{\omega} \times X_{q}^{\omega}$ may be rewritten as $\left(X_{p} \times X_{q}\right)^{\omega}$. Since the $\pi$-weight of $X_{p} \times X_{q}$ is $c^{+}$, and $X_{p} \times X_{q}$ is not pseudo- $c^{+}$-compact, it follows that $\left(X_{p} \times X_{q}\right)^{\omega}$ is a $\sigma-\pi$ space $\left[\mathbf{P}_{3}, 2.4\right]$.

\subsection{LEMMA. The product $X_{p}^{\omega} \times X_{q}^{\omega}$ has remote points.}

PROOF. Since $X_{p}^{\omega} \times X_{q}^{\omega}$ is a nonpseudocompact, $\sigma-\pi$ space, it must have remote points [H, 3.1].

5.11 EXAMPLE. There exist nonpseudocompact spaces $X$ and $Y$, each having no remote points, such that $X \times Y$ has remote points. (Furthermore, neither $X$ nor $Y$ is $\sigma-\pi$, but $X \times Y$ is.)

PROOF. Let $X=X_{p}^{\omega}$ and let $Y=X_{q}^{\omega}$.

6. Counterexample with no remote points. In this section additional set theoretic assumptions beyond ZFC are utilized. In order to present these assumptions some additional definitions are required.

6.1 DEFinition. Let ${ }^{\omega} \omega$ be the set of all functions from $\omega$ into $\omega$. If $f, g \in{ }^{\omega} \omega$, let $<^{*}$ be the partial ordering on ${ }^{\omega} \omega$ defined by $f<^{*} g$ if there exists $n<\omega$ such that for all $m>n, f(m)<g(m)$. Let $\Omega$ be the partial order $\left\langle{ }^{\omega} \omega,<^{*}\right\rangle$. A set $S \subset{ }^{\omega} \omega$ is said to be cofinal in $\Omega$ if for every $f \in{ }^{\omega} \omega$, there exists some $g \in S$ such that $f<^{*} g$. If the order type of a cofinal set is that of an ordinal $\eta$, then we shall call the set an $\eta$-scale.

It is well known that the set theoretic hypotheses "there exists, for each $j<\omega$, a compact Souslin line $S_{j}$ such that $\prod_{j<\omega} S_{j}$ is ccc" and "there exists an $\omega_{2}$-scale" are consistent with ZFC and, moreover, that there exists a model containing ZFC which satisfies both hypotheses simultaneously (see [KT]). Let $\mathscr{M}$ be any such model of set theory. (For more information on scales, see Heckler $[\mathbf{H}]$ or Rudin [R].)

The following theorems are significant tools for the construction of the counterexample.

6.2 THEOREM (DOW $\left[\mathbf{D}_{4}\right]$ ). If $X$ is a nonpseudocompact ccc space with $\pi X=$ $\omega_{1}$, then $X$ has remote points.

6.3 THEOREM (DOW $\left[\mathrm{D}_{5}\right]$ ). Assume that there exists an $\omega_{2}$-scale. If, for each $j<\omega, Z_{j}$ is a compact space of uncountable cellularity, then $\omega \times \prod_{j<\omega} Z_{j}$ has no remote points.

The counterexample depends upon the model $\mathscr{M}$.

6.4 COUNTEREXAMPLE (ZFC $\left.{ }^{+}\right)$. There exist nonpseudocompact spaces $X$ and $Y$, each having remote points, such that $X \times Y$ has no remote points.

\footnotetext{
${ }^{4}$ Lemmas 5.5 and 5.9 show that $X \times Y$ may be $\sigma-\pi$ even when neither $X$ nor $Y$ is. This provides an affirmative answer to Question 4.2 as posed in $\left[\mathbf{P}_{3}\right]$. This example may be compared to an example of $[\mathbf{T}]$ relative to a similar situation for $\sigma$-disjoint $\pi$-bases.
} 
ProOF. Let $X=Y=\omega \times \prod_{j<\omega} S_{j}$. Then $X$ and $Y$ have remote points by Theorem 6.2. However, $X \times Y=X^{2}$ and $X^{2}$ is homeomorphic to $\omega \times \prod_{j<\omega}\left(S_{j}\right)^{2}$, which does not have remote points by Theorem 6.3 since, for each $j<\omega,\left(S_{j}\right)^{2}$ is a compact space with uncountable cellularity.

ACKNOWLEDGMENTS. The authors acknowledge, with appreciation, the mathematical stimulus of W. W. Comfort and J. van Mill, regarding many of the problems considered in this research. The authors also express their appreciation to the referee for an attentive reading of the manuscript and for the constructive criticism towards its improvement.

\section{REFERENCES}

[CS] S. B. Chae and J. H. Smith, Remote points and G-spaces, Topology Appl. 11 (1980), 243-246.

[CN $\mathbf{C N}_{1}$ W. W. Comfort and S. Negrepontis, The theory of ultrafilters, Springer-Verlag, Berlin and New York, 1974.

[CN2] Chain conditions in topology, Cambridge Univ. Press, Cambridge, 1982.

[vD] E. K. van Douwen, Remote points, Dissertationes Math. 183 (1982).

[vDvM] E. K. van Douwen and J. van Mill, Spaces without remote points, Pacific J. Math. 105 (1983), 69-75.

[D1] A. Dow, Remote points in large products, Topology Appl. 16 (1983), 11-17.

[D2] _ Some separable spaces and remote points, Canad. J. Math. 34 (1982), 1378-1389.

[Ds] Weak P-points in compact ccc $F$-spaces, Trans. Amer. Math. Soc. 269 (1982), 557-565.

[D4], Remote points in spaces with $\pi$-weight $\omega_{1}$, Fund. Math. 124 (1984), 197-205.

[D5] _ _ Products without remote points, Topology Appl. 15 (1983), 239-246.

[D6] _ A separable space with no remote points, to appear TAMS.

[DP] A. Dow and T. J. Peters, Game strategies yield remote points, Topology Appl. 27 (1987) 245-256.

[FG] N. J. Fine and L. Gillman, Remote points in $\beta R$, Proc. Amer. Math. Soc. 13 (1962), 29-36.

[GJ] L. Gillman and M. Jerison, Rings of continuous functions, Springer-Verlag, Berlin and New York, 1976.

[H] S. H. Heckler, On the existence of certain cofinal subsets of ${ }^{\omega} \omega$, Axiomatic Set Theory, Proc. Sympos. Pure Math., vol. 13, part 2, Amer. Math. Soc., Providence, R.I., 1974, pp. 155-173.

[HP] M. Henriksen and T. J. Peters, Locally finite families, completely separated sets and remote points, Proc. Amer. Math. Soc. 103 (1988), 989-995.

[KM] A. Kanamari and M. Magidor, Higher set theory, Lecture Notes in Math., vol. 669 (G. H. Mulley and D. Scott, eds.), 1978, pp. 99-275.

[K] K. Kunen, Set theory, an introduction to independence proofs, North-Holland, Amsterdam, 1980.

[KT] K. Kunen and F. D. Tall, Between Martin's axiom and Souslin's hypothesis, Fund. Math. 102 (1979), 173-181.

[vM] J. van Mill, More on remote points, Rapport 91, Wiskundig Seminarium, Free University of Amsterdam, 1979.

[P1] T. J. Peters, Remote points, products and G-spaces, Doctoral Dissertation, Wesleyan Univ., 1982.

$\left[\mathbf{P}_{\mathbf{2}}\right]$, Dense homeomorphic subspaces of $X^{*}$ and of $(E X)^{*}$, Topology Proc. 8 (1983), 285-303.

$\left[\mathbf{P}_{\mathbf{3}}\right] \ldots$, For any $X$, the product $X \times Y$ has remote points for some $Y$, Proc. Amer. Math. Soc. 95 (1985), 641-648.

[R] M. E. Rudin, Lectures on set theoretic topology, CBMS Regional Conference Series in Math., No. 23, Amer. Math. Soc., Providence, R.I., 1975.

[T] S. Todorcevic, Stationary sets, trees and continuums, Publ. Inst. Math. 42 (1981), 249-262. 
[W] R. C. Walker, The Stone-Cech compactification, Springer-Verlag, Berlin and New York, 1974.

[Wi] S. Willard, General topology, Addison-Wesley, Reading, Mass., 1970.

Department of Mathematics, York University, North York, Ontario, CANADA M35 1P3 (Current address of A. Dow)

Computervision Corporation, Mail Code 14-2, 14 Crosby Drive, Bedford, MASSACHUSETTS 01730

Current address (T. J. Peters): Charles Stark Draper Laboratory, 555 Technology Square, Cambridge, Massachusetts 02139 